\title{
An Alternative Method to Obtain the Quark Polarization of the Nucleon
}

\author{
G. Igo \\ Department of Physics and Astronomy, UCLA, Los Angeles, California 90095
}

(October 6, 2000)

\begin{abstract}
An alternate method is described to extract the quark contribution to the spin of the nucleon directly from the first moment of the deuteron structure function, $g_{1}^{d}$. It is obtained without recourse to the use of input on the nucleon wave function from hyperon decays involving the flavor symmetry parameters, $\mathrm{F}$ and $\mathrm{D}$. The result for the quark polarization of the nucleon, $\Delta \Sigma_{N}$, is in good agreement with the values of the singlet axial current matrix element, $a_{0}$, obtained from recent next-to-leading order analyses of current proton, neutron and deuteron data.
\end{abstract}

PAC number(s): 13.60.Hb, 13.60.-r, 11.55.Hx

In polarized deep inelastic scattering experiments, the structure function, $g_{1}\left(x, Q^{2}\right)$, is measured. In leading order, it is the charge-weighted difference of the momentum distributions for quark helicities aligned and anti-aligned to the longitudinally polarized nucleon,

$$
g_{1}\left(x, Q^{2}\right)=\frac{1}{2} \sum_{i} e_{i}^{2}\left[q_{i}^{\uparrow}\left(x, Q^{2}\right)-q_{i}^{\downarrow}\left(x, Q^{2}\right)\right]=\sum_{i} e_{i}^{2} \Delta q_{i}\left(x, Q^{2}\right) .
$$

The total helicity $\Delta \Sigma$ due to the quarks, on the other hand, depends on $\int_{0}^{1} \Delta q_{i}\left(x, Q^{2}\right) d x=\Delta q_{i}$, the unweighted components of the spin of the nucleon carried by a quark of flavor $i$ and $\Delta \Sigma=\Delta u+\Delta d+\Delta s$. Thus to extract $\Delta \Sigma$ from the measurement of the first moment of $g_{1}\left(x, Q^{2}\right)$, additional axial vector matrix elements, derived with the assumption of the validity of isospin symmetry and SU(3) flavor symmetry, have often been used [1]]-[1]. This procedure has been questioned since $\mathrm{SU}(3)$ flavor symmetry-breaking effects are present and do not vanish for axial vector matrix elements. The situation is different for the first moment of the nucleon, $\Delta \Sigma_{N}$, defined as one-half 
of the sum of the neutron and proton helicities, in the quark parton model. The first moment of $g_{1}^{N}$ is directly proportional to $\Delta \Sigma_{N}$, within a small term proportional to $\Delta s$. Therefore it is possible to extract the former without a dependence on the $\mathrm{SU}(3)$ flavor symmetry parameters, F and D [5], [6]. In this paper it is shown that the two methods of extracting $\Delta \Sigma$, one in which the flavor symmetry parameters do not enter, and the other, the conventional approach, in which it is assumed that $\mathrm{SU}(3)$ symmetry-breaking effects can be neglected, yield values of $\Delta \Sigma$ in good agreement with one another. It should be pointed out that in the next-to-leading order QCD analysis [0] by the E154 Collaboration, the authors do not assume SU(3) flavor symmetry to fix the normalization of the non-singlet distributions by the axial charges $a_{3}=F+D$ and $a_{8}=3 F-D$. Their central ansatz of parameterizing the polarized parton distribution is that they are proportional to the unpolarized parton distributions obtained from ref. 88]. Another approach has been taken by Ohlsson and Snellman [9], who employ the broken SU(3) symmetric chiral quark model [10] to analyze the nucleon quark spin polarization measurements directly, without using any SU(3) symmetry assumption. This was accomplished by fixing the symmetry-breaking parameters of the model with measurements by the NuSea Collaboration [11], [12] of the quark sea isospin asymmetries. They obtain a value for $\Delta \Sigma\left(Q^{2}\right)$ of $0.29 \pm 0.14$ at $Q^{2}=5 \mathrm{GeV}^{2}$, in reasonable agreement with the summary of values shown in Figure 1 below.

The first moment of the nucleon, defined as one-half of the sum of the first moments of the proton and neutron spin structure functions, is expressible in terms of the first moment of the deuteron, $\Gamma_{1}^{d}$, and alternately, in terms of the axial currents, $a_{0}$ and $a_{8}$ [13]:

$$
\Gamma_{1}^{N}=\Gamma_{1}^{d} /\left(1-\frac{3}{2} \omega_{D}\right)=\int_{0}^{1} g_{1}^{d}\left(x, Q^{2}\right) d x /\left(1-\frac{3}{2} \omega_{D}\right)=\left(\frac{1}{36} a_{8} C_{n s}+\frac{1}{9} a_{0} C_{s}\right) .
$$

Here $\omega_{D}$ is the D-state probability of the deuteron and the quantities $C_{n s}$ and $C_{s}$ are $Q^{2}$-dependent coefficient functions. In the case of the proton (neutron) there is additional dependence on the axial current, $a_{3}$; i.e., for the proton:

$$
\Gamma_{1}^{p}\left(Q^{2}\right)=\int_{0}^{1} g_{1}^{p}\left(x, Q^{2}\right) d x=\left(\frac{1}{12} a_{3}+\frac{1}{36} a_{8}\right) C_{n s}+\frac{1}{9} a_{0} C_{s} .
$$

If polarized gluons do not contribute to the nucleon spin, the singlet and non-singlet matrix elements of the axial current are expressible in terms of 
$\Delta u, \Delta d$ and $\Delta s$. Assuming isospin invariance and $\mathrm{SU}(3)$ flavor symmetry, they can also be expressed in terms of the weak hyperon decay constants, $\mathrm{F}$ and $\mathrm{D}$, as

$$
\begin{gathered}
a_{0}=\Delta u+\Delta d+\Delta s=\Delta \Sigma, \\
a_{3}=\Delta u-\Delta d=F+D
\end{gathered}
$$

and

$$
a_{8}=\Delta u+\Delta d-2 \Delta s=3 F-D .
$$

The non-singlet coefficient function $C_{n s}$ and the singlet coefficient function $C_{s}$ appearing in Equations 2 and 3 have been calculated in the modified minimal subtraction $\overline{M S}$ scheme [14, [15] to third order for three quark flavors. The non-singlet coefficient is found to be,

$$
C_{n s}=1-\frac{\alpha_{s}\left(Q^{2}\right)}{\pi}-3.58\left(\frac{\alpha_{s}\left(Q^{2}\right)}{\pi}\right)^{2}-20.22\left(\frac{\alpha_{s}\left(Q^{2}\right)}{\pi}\right)^{3} .
$$

where $\alpha_{s}\left(Q^{2}\right)$ is the strong coupling constant. The singlet coefficient function $C_{s}$ has been evaluated in two forms in the $\overline{M S}$ scheme, one of which yields a $Q^{2}$-dependent $a_{0}$ in equations 2 and 3 and one, used below, which provides the high $Q^{2}$ limit to $a_{0}$ (The conclusions reached below are not affected substantially by this choice), is

$$
C_{s}=1-0.3333 \frac{\alpha_{s}\left(Q^{2}\right)}{\pi}-0.5495\left(\frac{\alpha_{s}\left(Q^{2}\right)}{\pi}\right)^{2} .
$$

In the $\overline{M S}$ scheme, gluons do not contribute to the first moment of $g_{1}$ and $a_{0}=\Delta \Sigma$, and the former is independent of the factorization scheme. In the Adler-Bardeen (AB) scheme [16, the invariant quantity, $a_{0}$ is not equal to $\Delta \Sigma$, and because of the axial anomaly [17] also involves the gluon spin structure function, $\Delta G\left(Q^{2}\right)$,

$$
a_{0}=\Delta \Sigma-\frac{3}{2 \pi} \alpha_{s}\left(Q^{2}\right) \Delta G\left(Q^{2}\right) .
$$

The values of $a_{0}$, extracted from the analysis by the SMC Collaboration [[]] of the world data in the two schemes, are consistent with one another 
within their experimental errors. In the extraction of $a_{0}$, from the world data for proton, deuteron and neutrons, the normalization of the non-singlet quark densities are fixed using the neutron and hyperon $\beta$-decay constants and assuming $\mathrm{SU}(3)$ flavor symmetry in the SMC analysis. The relation between the matrix element $a_{3}$, and the neutron $\beta$-decay constants, $g_{A} / g_{V}=$ $F+D$, relies only on the assumption of isospin invariance. However in order to relate $a_{8}$ to the semi-leptonic hyperon decay constants, F and D, $\mathrm{SU}(3)$ flavor symmetry is assumed and hence conclusions on $a_{0}$ depend on its validity. SU(3) symmetry-breaking effects do not vanish at first order for axial vector matrix elements [18]. It has been suggested that in order to reproduce the experimental values of $\mathrm{F}$ and $\mathrm{D}$, the quark-parton model requires large relativistic corrections which depend on the quark masses. Since the s-quark mass is much larger than that of $u$ and $d$ quarks, these corrections should break SU(3) symmetry. Similarly, the relations between the baryon magnetic moments predicted by $\mathrm{SU}(3)$ are badly broken [19]. The uncertainty in $a_{8}$ propagates into $a_{0}$. For instance, the value obtained for $a_{8}$ from a leading-order $1 / N_{c}$ expansion is much smaller that the value based on the SU(3) analysis. The use of a smaller value of $a_{8}$ causes $a_{0}$ to become larger [20], [9]. Symmetry breaking effects have been calculated under various assumptions leading to variations in the quantity $3 \mathrm{~F}-\mathrm{D}$ differing by a factor of two [21]-25]. Lipkin [26] criticizes the use of SU(3) symmetry in extracting the singlet axial current from the data, and maintains that the use of $F$ and D can only be considered fudge factors with no physical basis that should work for the axial current.

In view of the reservations discussed in the last paragraph about the use of the $\mathrm{SU}(3)$ flavor symmetry parameters in the extraction of $a_{0}$, it is of interest to compare with the results from the alternate method described here. As noted above, in the quark-parton model, the first moment $\Gamma_{1}^{N}$ of the nucleon structure function, $g_{1}^{N}=\frac{g_{1}^{p}+g_{1}^{n}}{2}$, can be expressed, up to a small correction factor depending on $\Delta s$, wholly in a term proportional to $\Delta \Sigma$, i.e. expressible without invoking $\mathrm{SU}(3)$ symmetry and as a consequence, $\mathrm{F}$ and D. We write 


$$
\begin{aligned}
\Gamma_{1}^{N} & =\Gamma_{1}^{d} /\left(1-\frac{3}{2} \omega_{D}\right)=\frac{1}{36}\left[(\Delta u+\Delta d-2 \Delta s) C_{n s}+4(\Delta u+\Delta d+\Delta s) C_{s}\right] \\
& =\frac{1}{36}\left[\left(C_{n s}+4 C_{s}\right) \Delta \Sigma\right]-\frac{1}{12} C_{c s} \Delta s
\end{aligned}
$$

Neglecting the last term in Equation 10 for the moment,

$$
\Delta \Sigma_{N}=36 \Gamma_{1}^{d} /\left[\left(C_{n s}+4 C_{s}\right)\left(1-\frac{3}{2} \omega_{D}\right)\right],
$$

thus expressing $\Delta \Sigma_{N}$ without explicit dependence on the $\mathrm{F}$ and $\mathrm{D}$ parameters. In Figure 1, $\Delta \Sigma_{N}$ at $Q^{2}=5 \mathrm{GeV}^{2}$ is plotted using the values for $\Gamma_{1}^{d}$ obtained by the E155 Collaboration at $5 \mathrm{GeV}^{2}$ [27] and at $10 \mathrm{GeV}^{2}$ by the SMC Collaboration [28]. These are in excellent agreement with the analysis of the world data for $a_{0}$ at $Q^{2}=5 \mathrm{GeV}^{2}$ by the E154 Collaboration [7] using the $\overline{M S}$ scheme and the $\mathrm{AB}$ scheme as illustrated in Figure 1. In the former scheme $a_{0}$ is equal to $\Delta \Sigma$; in the $\mathrm{AB}$ scheme, by Equation 9 . Figure 1 also gives the values of $a_{0}$ obtained by the SMC Collaboration in their analysis

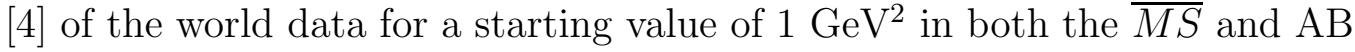
schemes. These are also in good agreement with the values of $\Delta \Sigma_{N}$ obtained in the analysis described in this paper. Inclusion of the term dependent on $\Delta s$ in equation 10 reduces the values of $\Delta \Sigma_{N}$, plotted in Figure 1 by approximately one-half of their experimental errors when $\Delta s$ is set equal to -0.08, consistent with the analysis by the E143 Collaboration [29] of their data. They obtained values for $\Delta s$ of $-0.08 \pm 0.03,-0.10 \pm 0.03,-0.07 \pm 0.04$.

In summary, it is shown in this note that the singlet axial current matrix element, $a_{0}$, obtained by the E154 [7] and the SMC [4] Collaborations in their respective analyses of the world data on proton, neutron and deuteron structure functions with the latter using $\mathrm{SU}(3)$ flavor asymmetry and the parameters $\mathrm{F}$ and $\mathrm{D}$ are quite consistent with $\Delta \Sigma_{N}$, obtained directly from the deuteron spin structure functions without the assumption of $\mathrm{SU}(3)$ flavor symmetry. This result provides further substantiation that the observed discrepancy shown in Figure 11 of the naive QPM prediction for $a_{0}$ [30] and experiment is not an artifact due to a significant breakdown of $\mathrm{SU}(3)$ flavor symmetry. 


\section{Acknowledgements}

This work was supported by the U.S. Department of Energy. I would like to thank S. Trentalange for a careful reading of the manuscript.

\section{References}

[1] M. Gluck et al., Phys.Rev. D53 (1996) 4775.

[2] R. D. Ball, S. Forte and G. Ridolfi, Phys. Lett. B378 (1996) 255.

[3] T. Gehrmann and W. J. Stirling, Phys. Rev. D53 (1996) 6100.

[4] B. Adeva et al., Phys. Rev. D58 (1998) 112002.

[5] F. E. Close and R. G. Roberts, Phys. Lett. B316 (1993) 165.

[6] C. Caso et al., Eur. Phys. Jour. C3 (1998) 1.

[7] K. Abe et al., Phys. Lett. B405 (1997) 180.

[8] M. Gluck, E. Reya and A. Vogt, Z. Phys. C6 (1995) 433.

[9] T. Ohlsson and H. Snellman, Eur. Phys. J., C7 (1999) 501.

[10] A. Manohar and H. Georgi, Nucl. Phys. B234 (1984) 189.

[11] E.A. Hawker et al, Phys. Rev. Lett. 80 (1998) 3715.

[12] J.C. Peng et al, Phys. Rev. D58 (1998) 092004.

[13] E.V. Shuryak and A.I. Vainshtein, Nucl. Phys. B201 (1982) 141.

[14] S. A. Larin and J. A. M. Vermaseren, Phys. Lett. B259 (1991) 345.

[15] S. A. Larin, Phys. Lett. B334 (1994) 192.

[16] R. D. Ball, S. Forte and G. Ridolfi, Nucl. Phys. B444 (1995) 287; ibid B449 (1995) 680(E); Phys. Lett. B378 (1996) 255. 
[17] S. L. Adler, Phys. Rev. 177 (1969) 2426; J. S. Bell and RF. Jackiw, Nuovo Cimento 47 (1969) 61; A. V. Efremov and O. V. Teryaev, Dubna Report No. JIN-E2-88-287 (1988); G. Altarelli and G. Ross, Phys. Lett. B212 (1988) 391; R. D. Carlitz, J. D. Collins and A. H. Mueller, ibid (1988) 214, 219.

[18] J. M. Gaillard and G. Sauvage, Ann. Rev. Nucl. Part. Sci. 34 (1984) 351.

[19] Z. Dziembowski and J. Franklin, J. Phys. G17 (1991) 213.

[20] J.Dai et al, Phys. Rev. D53 (1996) 273.

[21] R. L. Jaffe and A. V. Manohar, Nucl. Phys. B337 (1990) 509.

[22] P. G. Ratcliffe, Phys. Lett. B242 (1990) 271; ibid B365 (1996) 383.

[23] B. F. Donoghue, B. R. Holstein and S. W. Klimt, Phys. Rev. D35 (1987) 934.

[24] B. Ehrnsperger and A. Schaefer, Phys. Lett. B348 (1995) 619.

[25] X. Song, J. S. McCarthy and H. J. Weber, Phys. Rev. D55 (1997) 2624.

[26] H. J. Lipkin, Phys. Lett. B214 (1988) 429.

[27] P. L. Anthony et al., Phys. Lett. B463 (1999) 339.

[28] B. Adeva et al., Phys. Rev. D58 (1998) 112001.

[29] K. Abe et al., Phys. Rev. D58 (1998) 112003.

[30] J. Ellis and R. L. Jaffe, Phys. Rev. D9 (1974) 1444; ibid. D10(1974) 1669. 


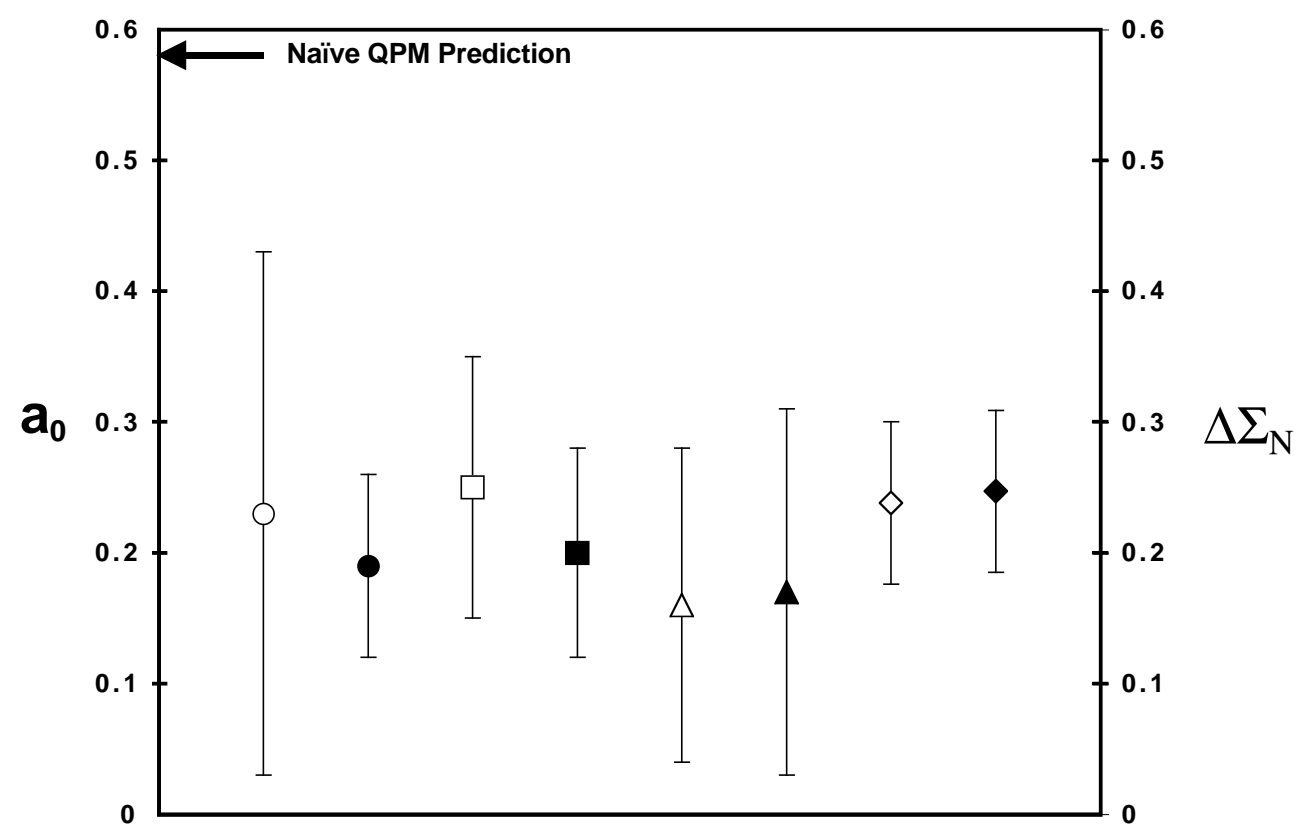

Figure 1: The singlet axial current matrix element, $a_{0}$, obtained from the analysis of world data by the SMC Collaboration[4] using the $\overline{M S}$ scheme (open circle), using the AB scheme (solid circle); same by the E154 Collaboration[7] using the $\overline{M S}$ scheme (open square), using the AB scheme (solid square). The quantity $\Delta \Sigma_{N}$ obtained from the first moments of the deuteron structure function measurement by the SMC Collaboration[23] (open triangle) and world data[4](solid triangle); same with measurements by the E155 Collaboration[22] using the low $x$ extrapolation of the E154 Collaboration[7] (open diamond) and using the low $x$ extrapolation of the SMC Collaboration[4] (solid diamond). 